\title{
Optical Communication Receiver Based on a Switched-Quadrature Costas Loop
}

\author{
A. Arvizu-Mondragón*1 ${ }^{* 1}$ F.J. Mendieta-Jiménez² ${ }^{2}$ J. de D. Sánchez-López ${ }^{3}$, I.Oropeza-Pérez ${ }^{4}$, J.R. López- \\ Leandro $^{5}$ \\ 1,2,3,4,5 Centro de Investigación Científica \\ y de Educación Superior de Ensenada (CICESE) \\ Carretera Ensenada-Tijuana No. 3918, Zona Playitas, \\ C.P. 22860 \\ Ensenada, B. C. México \\ arvizu@cicese.mx
}

\begin{abstract}
In this paper we present the development and implementation of a switched-quadrature optical Costas loop receiver and its performance evaluation by means of simulations and with the experimental work of an optical set up and electronic circuitry. We report as well the implementation of some of the basic building blocks that are required by both homodyne and heterodyne receivers for coherent optical communication systems (guided and unguided, i.e., optical fiber and free space optics): we present an automatic wavelength controller (AWC), an electronically-driven state of polarization controller (ASOPC) as well as an optical phase locked loop (OPLL) for phase tracking.
\end{abstract}

Keywords: phase tracking, switched-quadratures, optical Costas loop, optical phase locked loop, optical wavelength controller, state of polarization controller.

\section{RESUMEN}

En el presente trabajo reportamos el desarrollo e implementación de un receptor basado en un lazo de Costas óptico con cuadraturas conmutadas así como la evaluación de su desempeño mediante simulaciones y a través de un montaje experimental óptico y con circuitería electrónica. Reportamos asimismo la implementación de varios de los bloques básicos que son requeridos en los receptores ópticos homodinos y heterodinos con aplicación en sistemas de comunicaciones ópticas coherentes (guiados y no guiados, es decir, por fibra óptica y por espacio libre): presentamos el control automático de longitud de onda, el controlador del estado de polarización manejado electrónicamente así como el lazo de encadenamiento de fase (PLL) óptico.

\section{Introduction}

Recently there has been a renewed interest in the development, implementation and commercial deployment of homodyne, heterodyne and intradyne (guided and unguided) optical coherent communications systems for high capacity optical communications networks [1-10]. These systems have several well-known advantages over the more commonly used direct detection communication systems such as the possibility of compensating in the electronic domain several nonlinear impairments as well as the chromatic and polarization mode dispersions [5,11]. Additional advantages essential for coherent quantum receivers and for the free space optical channel, where there is no possibility of using in-line optical amplifiers, is the improved sensitivity $[2,5]$, and the possibility of using more efficient optical modulation formats (such as BPSK, POLSK QPSK, QAM, etc.) [12]. In addition, optical coherent detection enables the implementation of more densely packed wavelength division multiplexing systems to take advantage of the huge capacity of the optical channel [2,5]. To implement the optical coherent communication systems, several basic building blocks are needed such as an automatic wavelength controller in order to keep the frequency difference in the pass band of the photoreceiver and a dynamic state-of-polarization controller for homodyne and heterodyne systems both for the guided and unguided channels. In this 
paper, we describe such systems, their experimental implementation, as well as measurement of their operation characteristics.

\section{Tracking loops for optical phase processing}

Figure 1a shows the block diagram of a typical coherent optical homodyne communication system. Usually, on such kind of systems, the transmitter consists of a narrow linewidth laser source (operating in continuous wave) whose output is modulated (using an external optical modulator) and sent to the optical channel (optical fiber or free space). The receiver is implemented using an optical hybrid and several photoreceiver stages in a coherent balanced configuration. At the output of the photoreceivers, we get several electrical signals that may be used to recover both the electrical data and to control the wavelength, phase and state of polarization of the optical VCO required for the optical coherent detection (as it is described below).

In coherent optical communication systems, an optical phase synchronization (or optical phase estimation) stage is generally required in order to recover the electrical data. For homodyne receivers, a tracking loop such as an optical phase locked loop (OPLL) is commonly used for BPSK with residual carrier or an optical Costas loop for (the more power-efficient) BPSK with suppressed carrier (or optical QPSK). In optical heterodyne reception, similar loops are required at the intermediate (electrical) frequency but for both cases (homodyne and heterodyne), it is convenient to use a) an automatic wavelength controller (AWC) (see Figure 2a) to keep a minimum wavelength difference between the optical data signal and optical VCO for the adequate operation of the balanced photoreceiver stage; b) a state of polarization dynamic controller to get a more efficient optical mixing (see Figure 2a). In optical intradyne receivers, the local oscillator (usually a tunable laser oscillator) is in free-running operation, and the optical phase estimation and wavelength difference compensation are made over the (digitized) electrical observation signal with high-speed digital signal processing $[2,3,5,11,13]$.

In contrast, our work is focused on homodyne detection systems where several basic building blocks are required to implement an OPLL for residual carrier BPSK signals or an optical Costas loop for suppressed carrier BPSK signaling [14, 15]. In what follows, such building blocks are described.

\subsection{Balanced photoreception}

Figure $1 \mathrm{~b}$ shows a balanced photoreceiver consisting of a) an optical hybrid ( $\pi$ - hybrid or $\pi / 2$

- hybrid, depending on the type of modulation used) required to get the optical mixing of the local oscillator (optical VCO) and the optical data signal, b) $\mathrm{M}$ balanced photodetection stages (for M-ary modulations) to obtain the electrical observable signals needed to recover the data and for synchronization purposes at the homodyne and

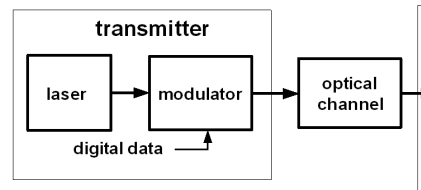

(a)

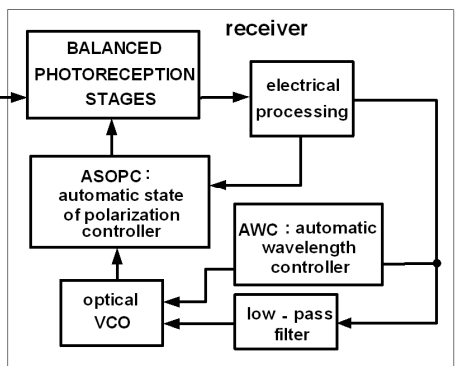

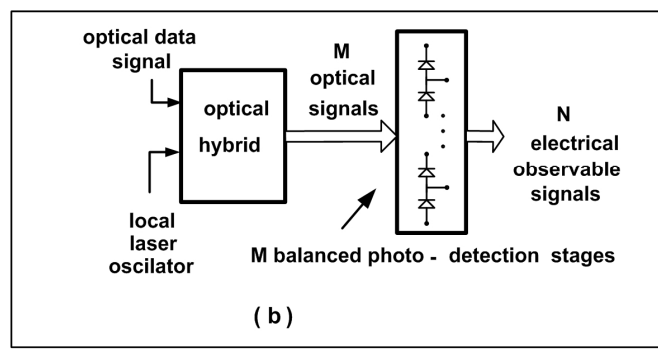

Figure 1. Block diagrams of: a) a homodyne coherent optical communications system, b) a balanced photoreception stage. 


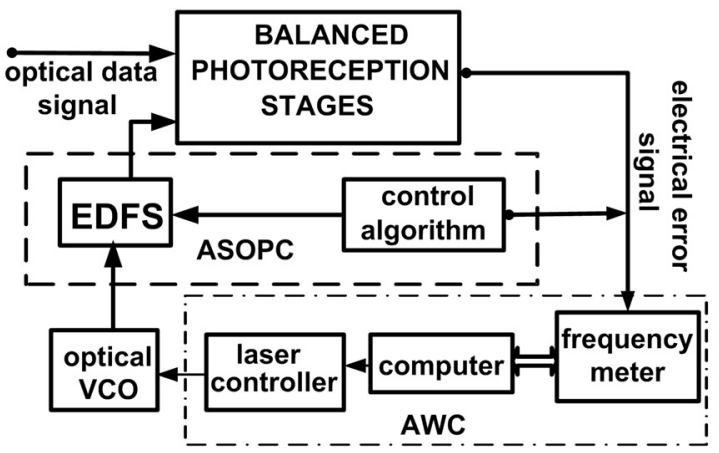

(a)

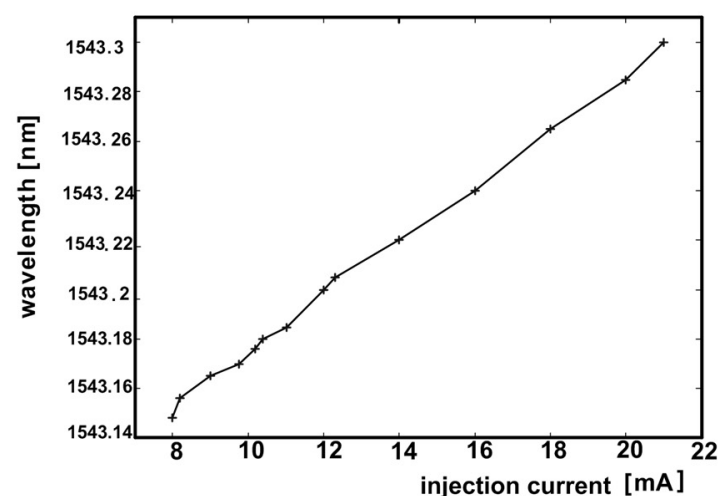

(b)

Figure 2. a) automatic controllers implemented: wavelength (AWC), state of polarization (ASOPC), b) measured wavelength injection current characteristic for the laser used in our experiments. EDFS: electronically driven fiber squeezer.

heterodyne receivers $[5,14]$. In our case, we implemented the balanced photodetection stage by means of the $\pi$ - hybrid (2x2 optical fiber coupler) whose electrical outputs are subtracted using highspeed amplifiers in a differential configuration to obtain the electrical observable signal used to recover the data and to extract the phase error to drive the AWC and ASOPC stages.

\subsection{Automatic wavelength controller (AWC).}

As previously mentioned, in optical coherent detection receivers (see Figure $2 a$ ), it is required to keep the wavelength difference between the optical data signal and the local oscillator constant (very close to zero and to the intermediate frequency for homodyne and heterodyne receivers respectively). Because of the relationship between the frequency and wavelength differences ( $\Delta f$ and $\Delta \lambda$, respectively), if any small change on the wavelengths is not compensated, the (electrical) frequency difference could be out of the electrical bandwidth of the photoreceiver stages (see Equation 1, where $\mathrm{c}$ is the speed of light).

$$
\Delta f=-\frac{c}{\lambda^{2}} \Delta \lambda
$$

Therefore, in optical coherent detection receivers, an important problem arises related to the precise adjustment on the optical VCO wavelength, due to the resolution in both the measurement systems and the electrical controller. For example, in our case, as we are using photoreceivers with an electrical bandwidth of $12 \mathrm{GHz}$ operating at $\lambda=1550 \mathrm{~nm}$ (193.414 THz), in order to remain operating on the electrical bandwidth of the photoreceiver, it is required to keep the wavelength difference within a value less than $0.096 \mathrm{~nm}$ (equivalent to $12 \mathrm{GHz}$ ). There exist several ways of controlling the wavelength of a laser, but for telecommunication applications where the distributed feedback (DFB) semiconductor lasers are widely used as optical sources, the wavelength may be controlled by actuating over its temperature and/or over its injection current $[14,18]$. In our case, we chose a DFB laser to implement an optical VCO; Figure $2 \mathrm{~b}$ illustrates the curve of its wavelength vs. injection current characteristic obtained experimentally. Based on the use of such laser, we implemented the homodyne receiver system of Figure $2 a$ consisting of a balanced photoreceiver stage whose electrical outputs are used for controlling both the state of polarization and wavelength of the optical VCO, and at the same time, it is used for demodulation purposes. Figure $3 a$ shows the automatic wavelength controller that we implemented taking advantage of the availability of a high resolution frequency meter and an ultra low-noise current source (a laser diode controller). 


\subsection{Automatic state of polarization controller (ASOPC).}

An optical signal transmitted through a standard optical fiber exhibits important fluctuations in its state of polarization (SOP) of the order of several $\mathrm{KHz}$ after hundreds of kilometers while for the same signal traveling through the unguided channel is on the order of hundreds of Hertz $[16,17,19]$.

The important issue of controlling the state of polarization of an optical field is commonly addressed by means of $\lambda / 4$ or $\lambda / 2$ polarizing plates (static control), Lefevre polarizers, fiber squeezers among others [18]. For our system, we implemented the dynamic SOP control in Figure 2a by means of an electrically-driven fiber squeezer (EDFS). Figure $3 b$ illustrates the block diagram of the electronic circuits designed to interface the EDFS with the observable signal and the controller.

\subsection{Switched-quadrature optical Costas loop (SQOCL).}

The OPLL is the more commonly used structure in coherent optical communications to synchronize and to recover the data when using phase modulation of the optical field [14, 15]. As more complex loops such as a conventional or a switched-quadrature optical Costas loops, (see Figures $4 a$ and $4 b$ ) may be built using the OPLL as a basic block, we have designed and implemented such a loop.

As seen in Figure 4a, a conventional optical Costas loop consists of a $\pi / 2$ optical hybrid with 2 optical inputs and four optical outputs that are used by two balanced photodetection stages to produce the in-phase (raw data signal) and quadrature electrical signals to be processed to obtain a phase error signal used to recover the suppressed (optical) carrier. The $\pi / 2$ optical hybrids have an inferior theoretical performance than the optical (lossless) $\pi$ - hybrids [15]. In addition, some of their practical implementations have adjustments and control signals that may lead to an error floor on the relative phase among the optical signals impinging on it [20].

To avoid these problems, we propose the use of a technique that we have called optical switched quadratures. This technique consists of producing the quadratures sequentially on time using just one branch. In order to do this, we switched the phase of the local oscillator between 90 and 0 degrees with the same bit period [22]. In the SQOCL structure in Figure 4b) the optical BPSK signal with suppressed carrier $E_{s}(t)$ (Equation 2a) is received at the input of an optical $\pi$-hybrid and mixed with a switched-quadrature optical laser oscillator signal $E_{S Q L O}(t)$ (Equation 2b).

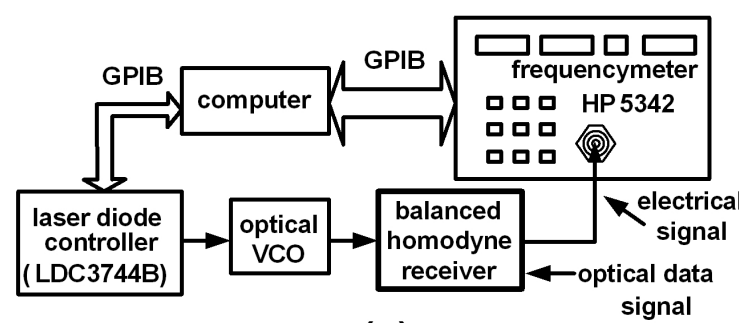

(a)

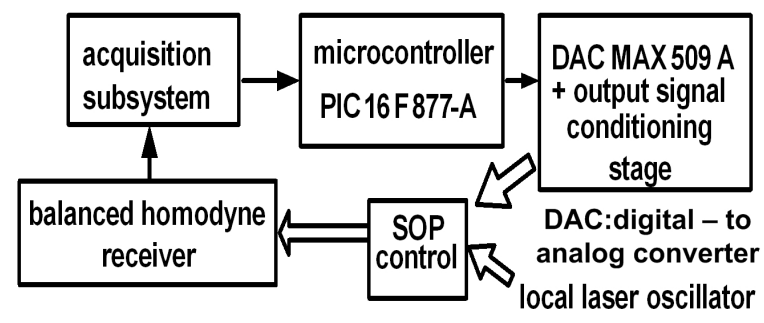

(b)

Figure 3. a) automatic wavelength controller implemented,

b) block diagram of the electronic circuits used the ASOPC. 

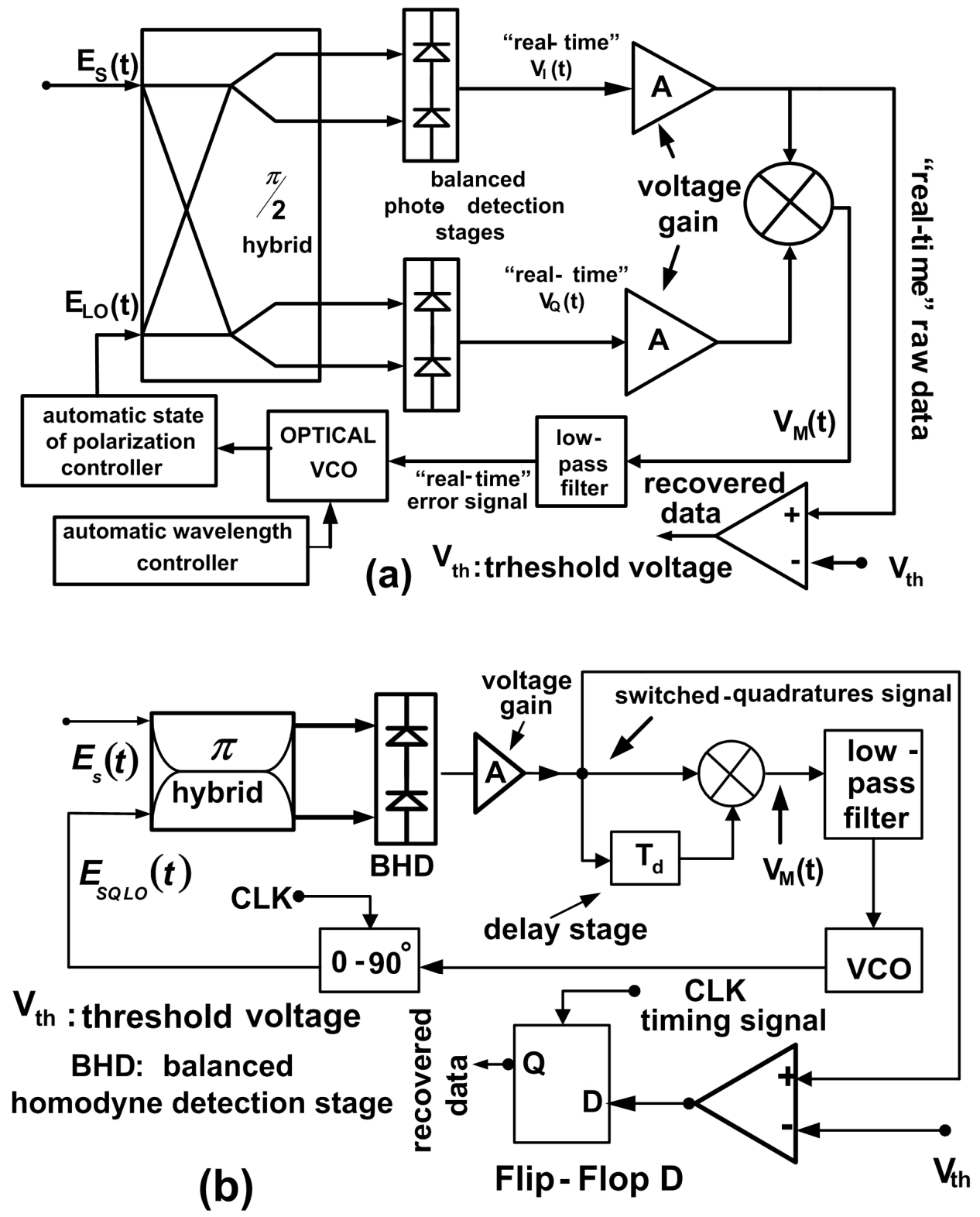

Figure 4. a) Conventional optical Costas loop,

b) Switched-quadratures optical Costas loop (SQOCL). 


$$
E_{s}(t)=x(t) \cos \left(2 \pi f_{s} t+\phi_{s}(t)\right)
$$

$$
E_{S Q L O}(t)=\cos \left(2 \pi f_{S Q L O} t+\phi_{S Q L O}(t)\right)
$$

where $\phi_{S}(t), f_{s}, \phi_{S Q L O}(t), f_{S Q L O}$ are the phase and frequency of the optical data signal and the local oscillator, respectively, $x(t) \varepsilon\left\{ \pm \frac{\pi}{2}\right\}$ is the data signal. The balanced photoreceiver stage at the output of the optical hybrid produces an electrical signal proportional to the square of the addition of the two optical fields and when the loop is operating in the phase-locked condition we obtain the error voltage signal:

$$
V_{e}(t)=\frac{r_{L} \Re P_{H} A}{2} \cos \left(\phi_{S}(t)-\phi_{S Q L O}(t)\right)
$$
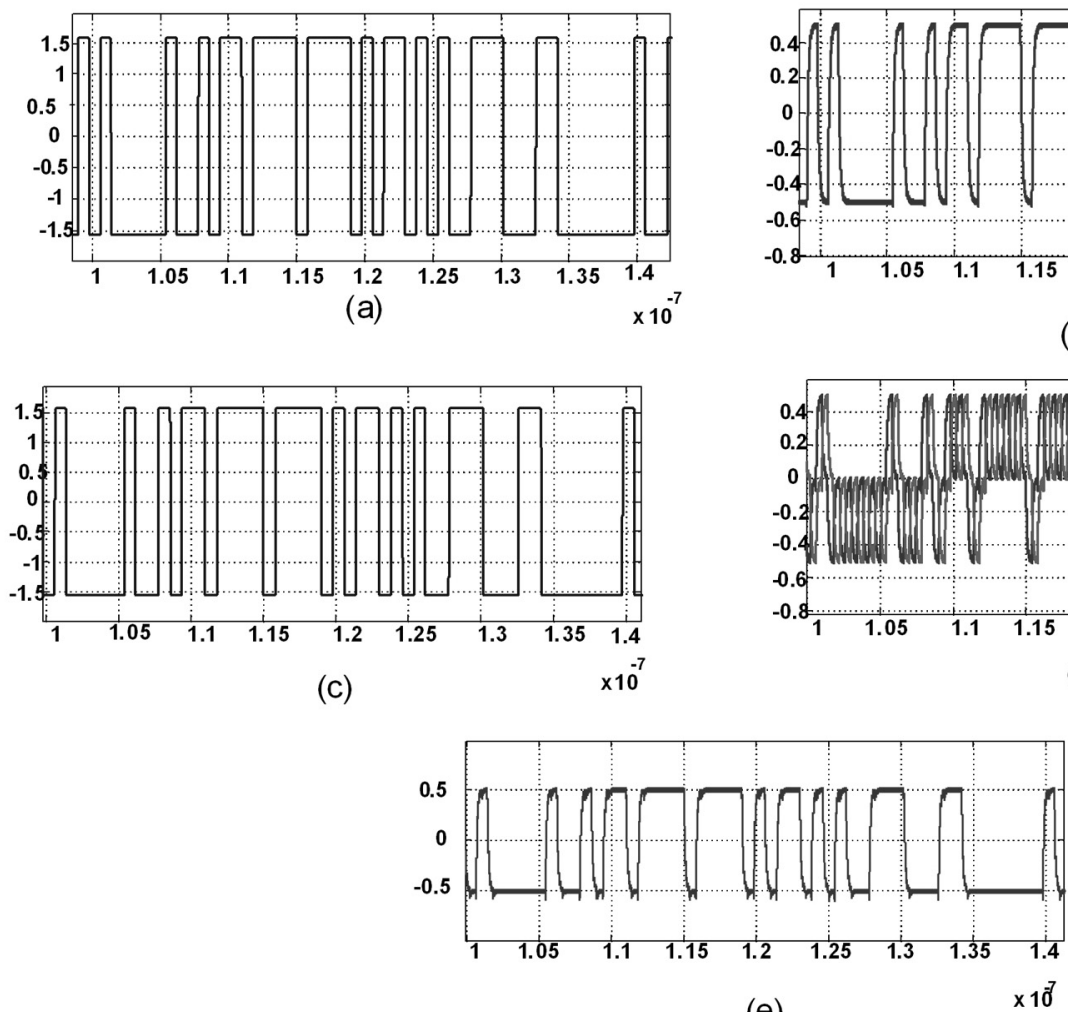

(e)

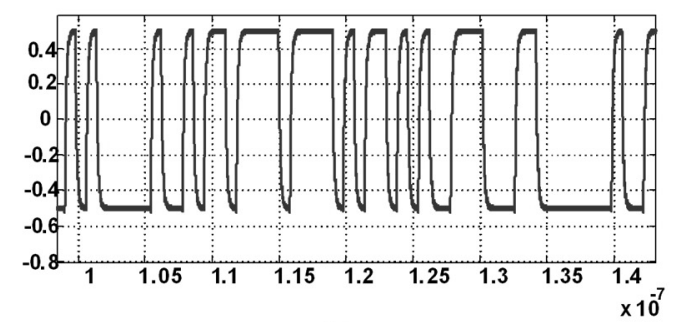

(b)

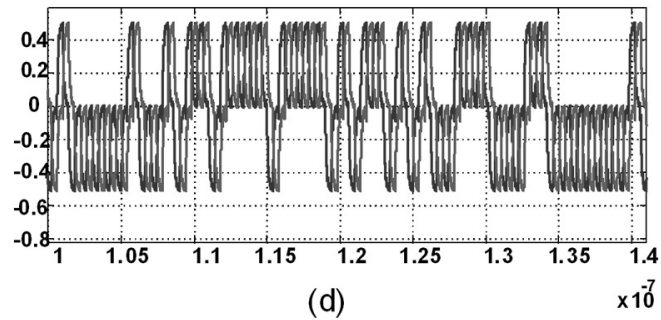

(d)

where $r_{L}$ : load resistance, $\mathfrak{R}$ : photodetector responsivity, $P_{H}$ : homodyne optical power, $A$ : photoreceiver gain.

In the conventional optical Costas loop (Figure 4a), we obtain two electrical signals using two balanced photoreception stages at the outputs of a $\pi / 2$ optical hybrid. Such signals are called the in-phase $\left(\mathrm{V}_{1}(\mathrm{t})\right)$ and quadrature $\left(\mathrm{V}_{\mathrm{Q}}(\mathrm{t})\right)$ components, due to their relative phase difference of 90 degrees. In our loop, we sequentially produce the in-phase and quadrature electrical signals mentioned above in order to operate in a similar fashion like a conventional (real-time) optical Costas loop. In order to suppress modulation, we multiply the signals obtained in this way to produce the signal $\left(V_{M}(t)\right)$ that has to be filtered to get the phase error

Figure 5. Operation of the a) Conventional optical Costas loop,

b) Switched-quadratures optical Costas loop, obtained by simulation. 
signal required to drive the optical VCO. The key for a good operation of the switched-quadrature loop is the synchronous optical phase-switching of the I-Q signals (using in our case is the timing signal (CLK), see Figure 4b). We have to include a delay stage $(\mathrm{Td})$ in order to get the signal $\mathrm{Vm}(\mathrm{t})$.

\section{Performance evaluation}

In order to demonstrate that the switchedquadrature optical Costas loop (SQOCL) is able to operate like a conventional (real-time) Costas loop, we have first simulated both loops using the Matlab and Simulink software and the more representative results are shown in Figure 5. For convenience, we make the following assumptions: a) a clock signal without jitter to switch the quadratures is available, b) both loops are operating on the phase-locked condition. The use of the last assumption implies that both loops are operating on their linear region (a usual simplification when analyzing such kind of loops).

For our simulations, we considered convenient to use "real world data signals" taken from a file of data acquired using a real-time oscilloscope. Figure 5a shows a sample of such data used to get BPSK modulation of the optical carrier to be sent in the optical channel while Figure $5 b$ shows the data recovered using the conventional optical Costas loop; Figure $5 \mathrm{c}$ shows a sample of the data used for simulating the SQOCL performance, while Figures 5d-e show the switched data and the recovered data using the SQOCL, respectively. Although, the switched data signal is obviously not the final data desired, we have included it in our reported results just to show the intermediate processing stage that does not exist in the realtime Costas loop.

As mentioned previously, a Costas loop is able to recover simultaneously the suppressed carrier and the data on a BPSK communication system. However, for simplicity, in our simulations we considered that we were operating on a phaselocked condition, i.e., the suppressed carrier had been recovered, so the only thing that we had to compare on both loops (the conventional and the switched-quadratures loops) was that the data were recovered adequately. As it is clearly shown in Figures $5 \mathrm{~b}$ and $5 \mathrm{e}$, both loops were able to recover the data, although, with the above- mentioned differences in their respective operation.

Also, we implemented a simplified electronic version of the optical SQOCL (without the data regeneration stage) as shown on Figures 6a-b, the data transmitted and the raw data signal, respectively (a "1010...." binary sequence) when an electrical carrier frequency $\left(F_{c}\right)$ of $100 \mathrm{MHz}$ and a bit rate $\left(B_{r}\right)$ of 2 Mbps were used; also in this case a lock-in range of $30 \mathrm{MHz}$ was measured. Commonly the raw data signal is used to get a regenerated data signal (using a digital regenerator) with a standard digital level (for example TTL, ECL, etc...). Although, at this development stage, we had not implemented such block, it can be seen from the available raw data signal (shown in Figure 6.b) that the information signal may be recovered from it through the use of digital regeneration stage improving the quality of such signal.

As mentioned above, we have considered very convenient to develop an OPLL because it is a basic structure needed to implement a switchedquadrature optical Costas loop. The methodology used to design such loop is based on the previous work of Kazovsky [15] and also on several works by the authors of the present paper. The main parameters that we have to consider on designing such loops are the bit rate, the optical transmitter and optical VCO line widths as well as the OPLL acquisition ranges required. Figure 7 shows the OPLL that we have designed and implemented operating at $1550 \mathrm{~nm}$ while Figure 8 shows a suggested implementation for the SQOCL.

Figure 9a shows a sample of sent and recovered raw data signal using the OPLL, respectively at a bit rate of 500 Mbps $\mathrm{nm}$ at $\mathrm{BER}<10^{-9}$ while Figure $9 \mathrm{~b}$ illustrates the eye diagrams obtained using a data pseudorandom binary sequence (to get an optical BPSK signal) and the recovered data at the output of the OPLL, respectively. Our measured OPLL lock-in range was $300 \mathrm{MHz}$.

On the kind of loops described above, a digital regeneration block is usually included in order to get a "useful" version of the data, i.e., a "cleaner" data signal without additive noise and with a standard digital level. The results shown in Figure 9 include the signals previous to such regeneration 

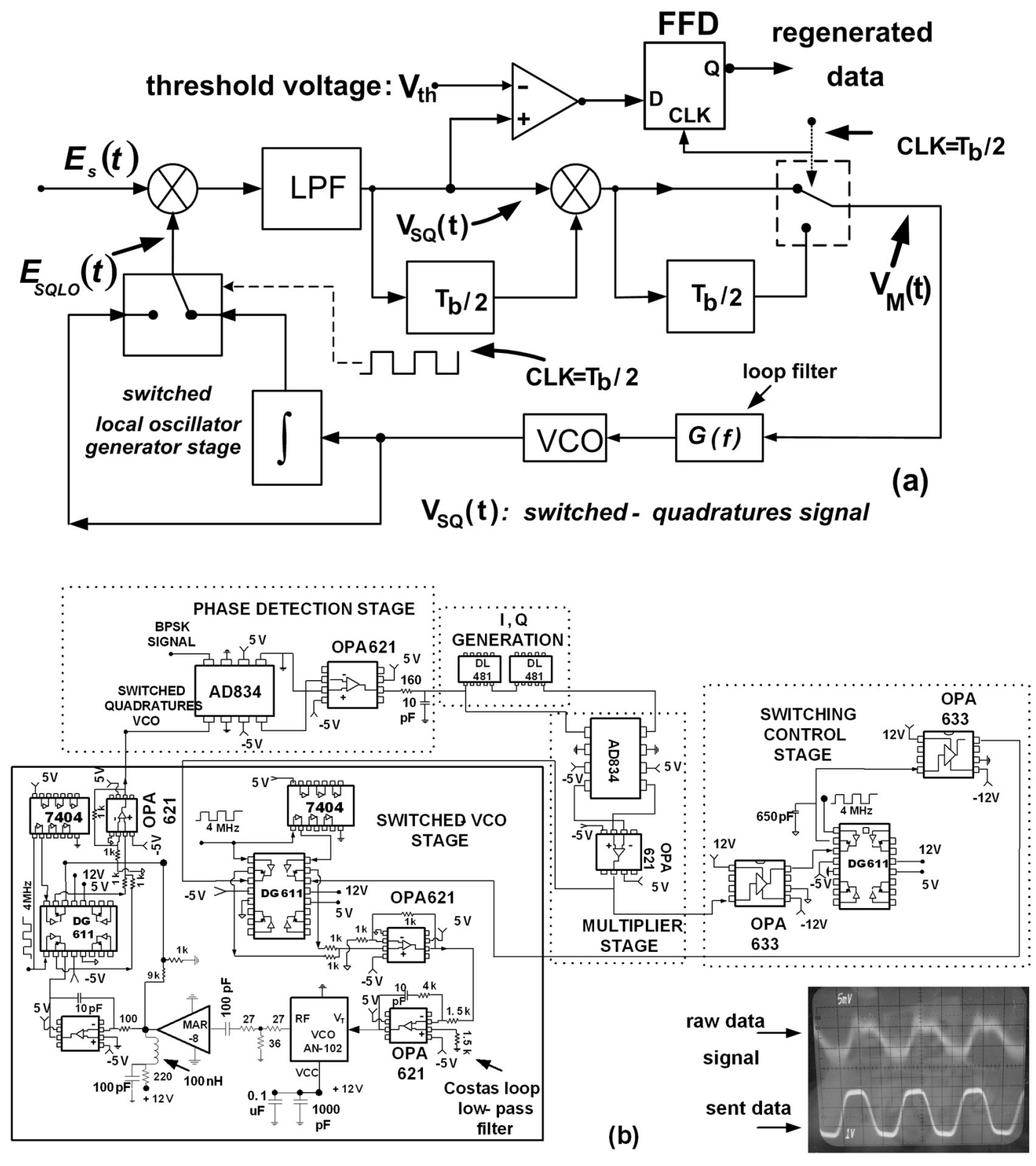

Figure 6. Proof-of-concept electronic version of the switched-quadratures optical Costas loop: a) schematic diagram, b) electrical diagram, with raw data signal and sent data respectively. 


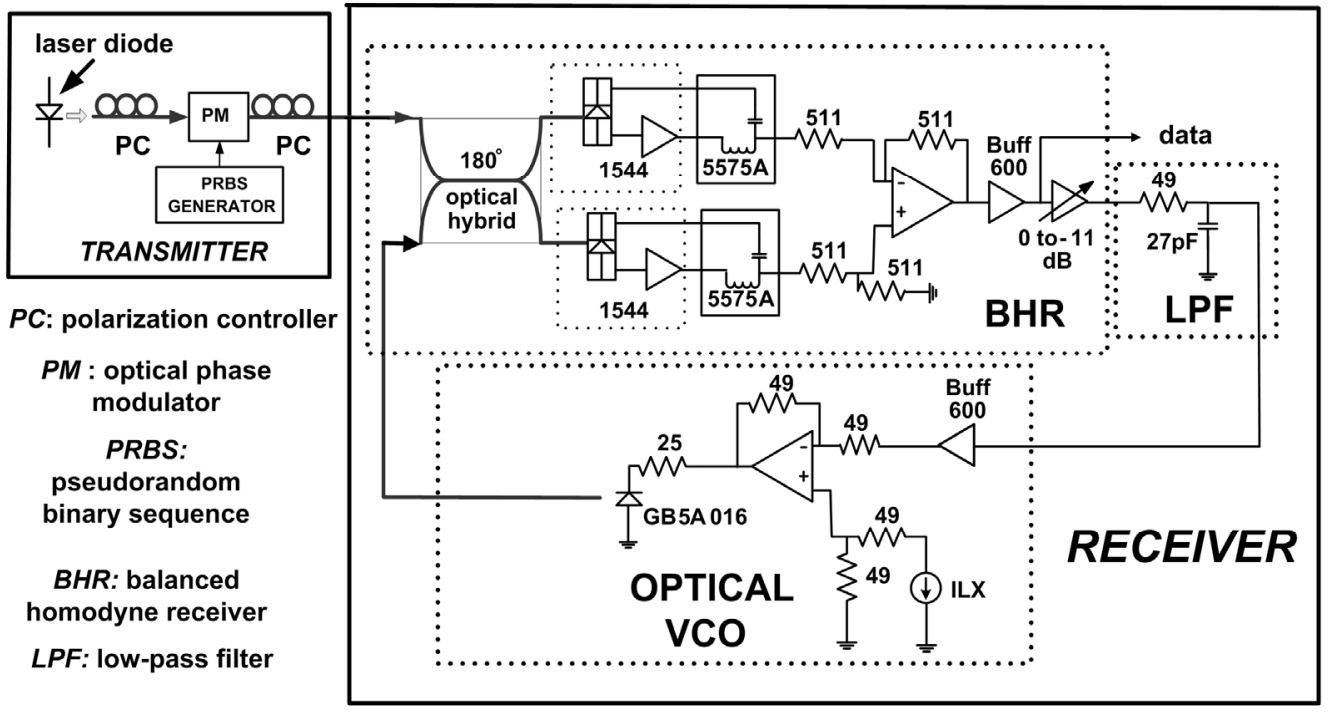

Figure 7. Implemented OPLL.

stage (the raw data signal); in such a case, the use of an eye diagram is very useful to evaluate the bit error rate expected after the regeneration stage, in our case, we measured a BER $<10^{-9}$ that it is a value commonly used for telecommunication purposes. Hence, we consider that our design may fulfill the telecommunications standard.

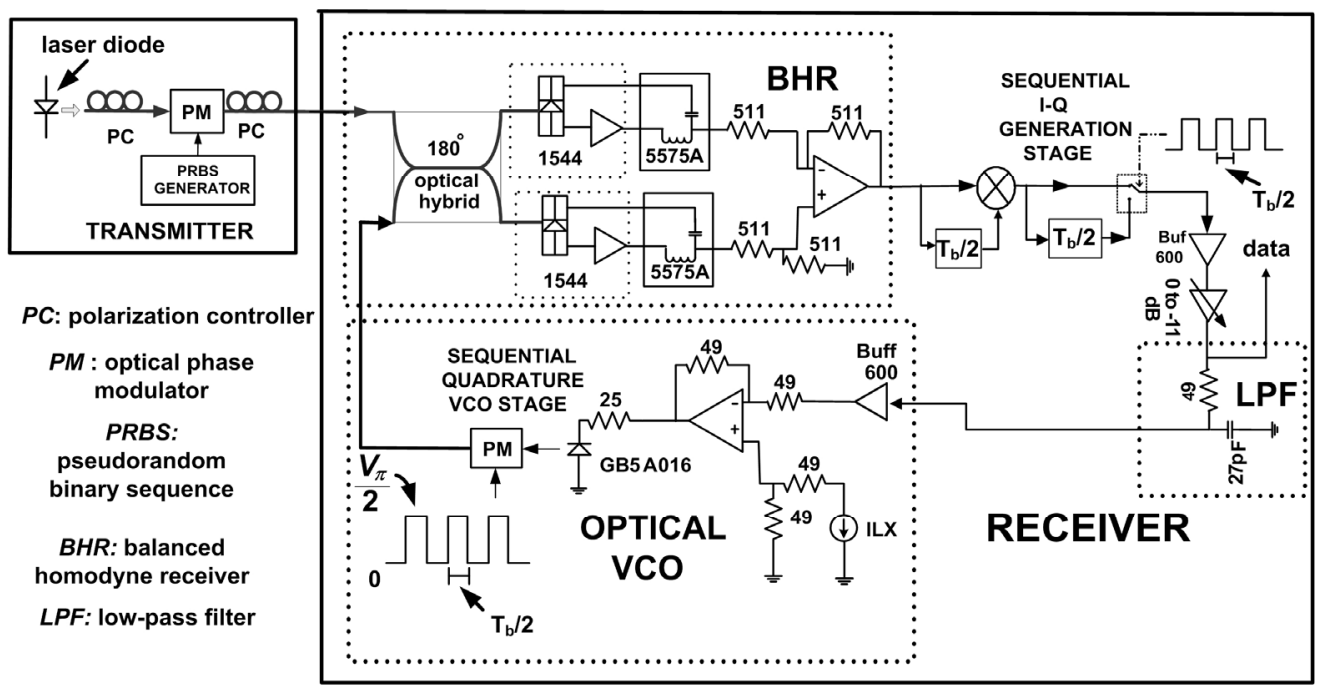

Figure 8. Suggested implementation for the SQOCL. 
Some of our results may be summarized as follows: a) we have designed and implemented a SOPC and AWC subsystems with an adequate performance for our OPLL (to operate on the phase-locked condition), b) we have developed and tested the simulation models for the conventional and switched-quadrature optical Costas loop in the phase-locked condition, c) we have designed, implemented and characterized an electronic version of the SQOCL that has been very useful as a proof-of-concept block, d) we have designed and implemented an OPLL with a good performance (BER $<10^{-9} @ 1550 \mathrm{~nm}, \mathrm{~B}_{\mathrm{r}}=500$ Mbps), that consists of several of the basic building blocks required to implement a SQOCL (that we are currently working on).

\section{Conclusions}

In this paper we have presented the development of a switched-quadrature optical Costas loop (SQOCL) and its performance evaluation by means of simulations and with the implementation of an optoelectronic system. We have developed as well an OPLL as a basic structure needed to implement a switched-quadrature optical Costas loop. The OPLL that we have designed and implemented operates at a bit rate of $500 \mathrm{Mbps}$ at $1550 \mathrm{~nm}$ wavelength with a BER $<10^{-9}$ and a lock- in range of $300 \mathrm{MHz}$. At the same time, for the OPLL implementation, we have designed and implemented as well an automatic wavelength controller, and an electronically-driven state of polarization controller. As it has been shown, the SQOCL has an equivalent operation as the realtime optical Costas loop with the advantage of not requiring the optical $\pi / 2$ - hybrid and two balanced homodyne receiver stages; the increase in speed and complexity is easy to trade off.

Currently, the theory, experimentation and field deployment of optical loops is an active field of research both for classical and quantum coherent communications. In this paper, we have presented several results in this field such as the feasibility of implementing a switched- quadrature optical Costas loop with readily available optical and optoelectronic components. At present, we are working on the implementation of such a loop both for classical and quantum states and in our future work we will test the SQOCL in the out-of-lock and in-lock conditions, measuring parameters such as the lock-in range, the holding range, and transient responses as well as the BER when several disturbances are considered such as the chromatic dispersion, on a long-distance high-speed communication link.

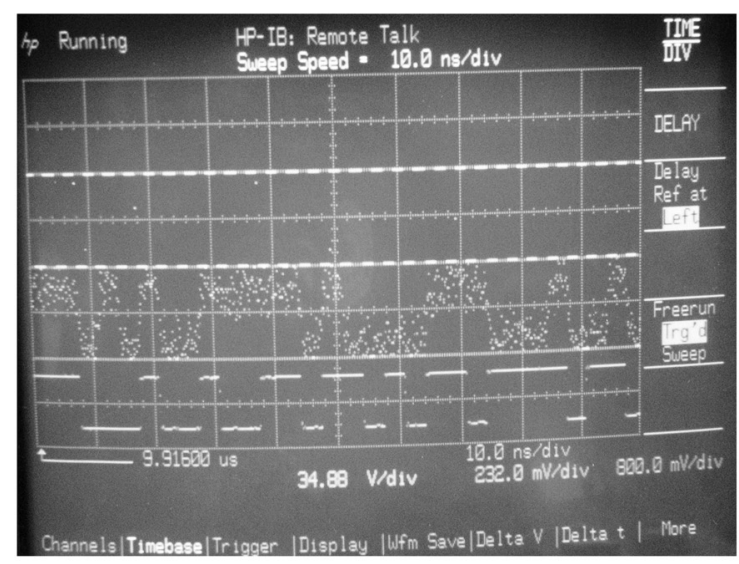

(a)

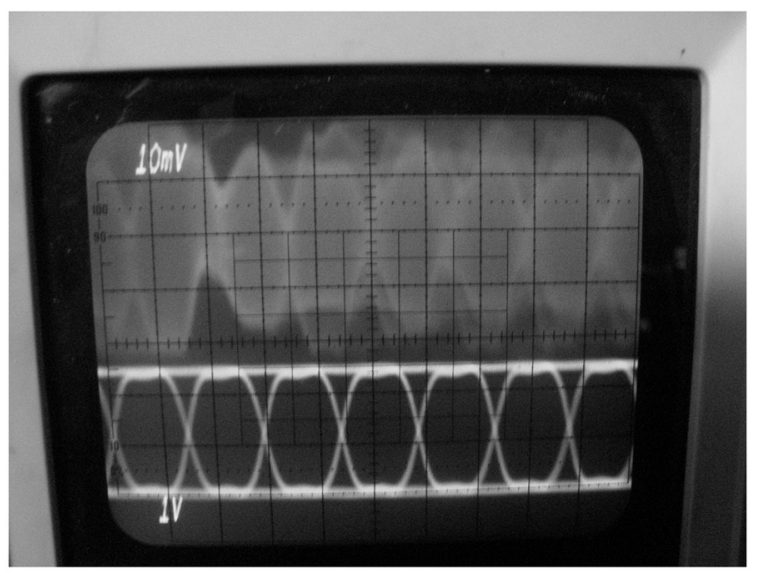

(b)

Figure 9. a) recovered and sent data using the OPLL respectively $(\mathrm{Br}=500 \mathrm{Mbps}$ operating at $1550 \mathrm{~nm}$ at BER<10-9), b) eye diagrams obtained using the recovered data at the output of the OPLL and a data pseudorandom binary sequence respectively. 


\section{References}

[1] N.Cvijetic et al, Polarization-multiplexed optical wireless transmission with coherent detection, IEEE Journ. of lightw. techn, Vol. 28, No.8, Ap. 15, 2010, pp.1218-1227.

[2] J.C.Rasmusen et al, Digital Coherent Receiver Technology for 100-GB/s optical Transport Systems, Fuj. Sci. Tech.J.,Vol.46,No.1, pp.63-71, Jan. 2010.

[3] X. Li et al, Electronic post-compensation of WDM transmission impairments using coherent detection and digital signal processing, Opt. Exp.16(2), 21 Jan. 2008, pp. $880-888$.

[4] Pfau T. et al, Towards Real-Time Implementation of Coherent Optical Communication, Opt. Exp., 2009, 16(2), pp. 866-872.

[5] Guifang L., Recent advances in coherent optical communications, Advances in Optics and Photonics. 1, 2009, pp. 279-307.

[6] A.Matiss et al, Novel Integrated Coherent Receiver Module for $100 \mathrm{G}$ Serial Transmission, Postd. Pap. PDPB3, OFC2010 Conf., San Diego, CA, USA, March $25,2010$.

[7] S. Zhang et al, Experimental demonstration of decision-aided maximum likelihood phase estimation in 8-channel 42.8-Gbit/s DWDM coherent PolMux-QPSK system, Session OMK1 OFC2010 Conf., San Diego, CA, USA, March 25, 2010.

[8] C. Fludger et al, Real-time prototypes for digital coherent receivers, Session OMS1 OFC2010 Conf., San Diego, CA, USA, March 25, 2010.

[9] T. Sakamoto et al, Digital optical phase locked loop for real-time coherent demodulation of multilevel PSQ/QAM, Session OMS5 OFC2010 Conf., San Diego, CA, USA, March 25, 2010.

[10] J. E. Simsarian et al, Fast-tuning $224 \mathrm{~Gb} / \mathrm{s}$ intradyne receiver for optical packet networks, Postd.Pap.PDPB5, OFC2010 Conf., San Diego, CA, USA, March 25, 2010.

[11]M.G. Taylor, Coherent detection method using DSP for demodulation of signal and subsequent equalization of propagation impairments, IEEE Phot.Techn.Lett.16(2), 2004,pp. 674-676.

[12] E. Ip et al, Coherent detection in optical fiber systems, 21 Jan. 2008 , Vol. 16, No. 2, Opt. Exp., pp.753-791.
[13] A.Leven et al Feed-forward phase and frequency estimation in coherent digital and analog photonic links using digital signal processing, IEEE/MTT-S 2007,3-8 June 2007, Honolulu, HI, pp.1511-1514.

[14]Keang-Po H.,Phase-modulated optical communications systems. Springer,2005,430 pp.

[15] Kazovsky et al, Optical fiber Communication Systems, Artech House, 1996, 690 pp.

[16] A.E. Willner et al, Monitoring and Control of Polarization-Related Impairments in Optical Fiber Systems, IEEE Journ.Lightw. Tecn., Vol. 22, No. 1, JAN.2004,pp.106-125.

[17] Koch B.et al, Endless Optical Polarization Control at $56 \mathrm{krad} / \mathrm{s}$, Over 50 Gigaradian, and Demultiplex of 112$\mathrm{Gb} / \mathrm{s}$ PDM-RZ-DQPSK Signals at $3.5 \mathrm{krad} / \mathrm{s}$, IEEE Journ. of. Sel. Top. in Quant. Elec., Sept.-Oct. 2010,Vol.16, Issue:5, pp. $1158-1163$.

[18] Franz, J. H., Jain,V.K,Optical communications, components and systems, CRC Press,. 2000, 717 pp.

[19] Andrews, L. C., Phillips, R.L. Laser beam propagation through random Media. SPIE Press, 2005, pp. 782.

[20] http://www.celight.com/images/pdfs/CL-QOH-90-42005.pdf

[21] Fabrega, J. M., Prat, J. Homodyne receiver prototype with time-switching phase diversity and feedforward analog processing, Opt. Lett., 32(5), 2007, pp. 463-465. 


\section{Authors' Biographies}

\section{Arturo ARVIZU-MONDRAGÓN}

He received the B.S.E.E. and M.Eng. degrees in electronics from Universidad Nacional Autónoma de México, UNAM, Mexico City, Mexico, in 1985 and 1990, respectively, and the Dr. Eng. degree in telecommunications from CICESE, Ensenada, B.C, Mexico, in 2000. In 1987, he joined the Institute for Electrical Research, IIE, Cuernavaca, Mexico, where he was engaged in projects related to the optical and optoelectronics communication systems with application in power generation systems, and laboratories for test, measurement, and characterization of electrical systems. In 1992, he joined CICESE, where he is currently involved in the fields of optical fiber and optical wireless communications with coherent detection and quantum communications.

\section{Francisco Javier MENDIETA-JIMÉNEZ}

He received the bachelor's degree in mechanical and electrical engineering from UNAM, Mexico City, Mexico; the master's and doctor's degrees from ENST, Telecom Paris Tech, Paris, France, in coherent optical communications. He was with the IIE, Mexico, where he was involved in the development of projects on applications of optical fibers to electrical power systems. He was with UNAM, where he was the leader of a continuous education program on telecommunications systems. During a leave at the Utah State University, USA, he participated in a project on instrumentation for space experiments. He formed the Optical Communications Group, and led several research projects on telecommunications and sensing at the CICESE, Ensenada, B.C., Mexico, where he is currently a lecturer for the Graduate Program on Optical and Digital Telecommunications, and has been an advisor for diverse master's and doctor's thesis. He has authored or coauthored diverse journal papers and conference papers in the field of optical communications and sensing.

\section{Juan de Dios SÁNCHEZ-LÓPEZ}

He received the B. Eng (Electrical Engineering) degree from Mechanical and Electrical Department of Technological Institute of Madero City in 1988. He received M.S. and Ph.D. degrees from the CICESE Research Center, Ensenada, Mexico in 1999 and 2010, respectively. Since 1994, he has been associate professor in the Department of Electronic Engineering of Universidad Autónoma de Baja California. His research interests include wireless communications, optical communications, digital signal processing and wireless sensor networks. 


\section{Israel OROPEZA-PÉREZ}

$\mathrm{He}$ received the $B$. Eng (electrical engineering) degree from the UAMIztapalapa, Mexico City, in 2006. He received the M.S. degree from CICESE Research Center, Ensenada B.C, Mexico in 2009. His research interests include optical and wireless communications and digital signal processing.

\section{Jesús Roberto LÓPEZ-LEANDRO}

He received the $B$. Eng (Electrical Engineering) degree from Instituto Tecnológico de Mazatlán, Sinaloa, Mexico, in 2006. He received the M.S. degree from the CICESE Research Center, Ensenada B.C, Mexico in 2009. $\mathrm{His}$ research interests include electronical instrumentation, control, digital signal processing and telecommunications. 